\title{
A Novel Front-End Radio Frequency Pressure Transducer based on a Dual-band Resonator for Wireless Sensing
}

\author{
Trang T. Thai ${ }^{1}$, Gerald R. DeJean ${ }^{2}$, and Manos M. Tentzeris ${ }^{1}$ \\ ${ }^{1}$ GEDC, School of ECE, Georgia Institute of Technology, Atlanta, GA 30332, U.S.A. \\ ${ }^{2}$ Microsoft Research, One Microsoft Way, Redmond, WA 98052, U.S.A
}

\begin{abstract}
A highly sensitive and compact radio frequency transducer is designed for differential pressure sensing that utilizes a stacked-patch resonator. This device, the first of its kind to be reported, functions as both a pressure sensing agent and a wireless communicating front-end, thus allowing miniaturization and easy integration. The resonator consists of a stacked-patch based on LTCC multilayer packaging technology, having an air cavity embedded by a silicon ( $\mathrm{Si}$ ) diaphragm that deflects due to pressure change. In one implementation, this transducer is designed to provide a communications link at $32 \mathrm{GHz}$ and a pressure sensing between 47-55 GHz. The pressure inside the air cavity can be calibrated to detect differential pressure anywhere from zero to several bars. In this paper, the authors present measurement results of the sensor prototype scaled to operate between approximately $5-8 \mathrm{GHz}$ as a proof-of-concept.
\end{abstract}

Index Terms - Pressure sensor, Radio Frequency transducer, dual-band, stacked patch, LTCC technology, resonator, RF integration.

\section{INTRODUCTION}

Pressure sensors are essential for controlling and monitoring thousands of everyday applications. They are employed in many fields such as medical technology (as in physiological monitoring), industrial uses (such as automotive control with measurements on fluid flow, acceleration, and displacement), and various military applications. Pressure transducers refer to devices that can transform the pressure change in the monitored environment into a change in the electrical signal. Common pressure transducer classes include resistive, piezoresistive, and capacitive based sensors, while others are based on inductive-capacitive circuits whose detection relies on resonant frequency shifts. Many of these classes utilize a diaphragm that deflects in response to pressure change, and can be used to measure a wide range of parameters such as air and liquid pressure as well as temperature in places where its change directly causes a change in pressure. Most transducers require physical wires to connect to an electronic device (to record and process information) and an antenna (in wireless applications to transmit or receive data). At the same time, wireless sensor networks require remote sensing enabled nodes that can be integrated into standard radio communication technologies. Furthermore, power consumption is an important factor that limits the functionality of the sensors. This problem is often alleviated by either operating the sensor on a short duty cycle or leaving the sensor in "stand-by" mode with a transceiver circuit to detect a "wake-up" signal [1]. Hence, there is a need to design a sensor that incorporates all of the features of high sensitivity, unobtrusive connectivity, and power consumption minimization in a single structure.

The new transducer presented here transforms the pressure change into a radio frequency (RF) signal shift. A previous pressure micro-sensor design was based on an RF transducer, reported in [2], which is itself a transmission line based filter and would require an antenna connection along with matching circuits for wireless sensing. On the contrary, the design presented in this paper is solely a pressure transducer, whose structure is defined by a resonator and functions both as a wireless communication link and a wireless differential pressure indicator. Thus, it eliminates not only the use of a signal processing circuit, but also the need for an antenna as the front-end component along with any matching circuit, therefore significantly simplifying and miniaturizing the complete sensor. The resonator operates in two bands between 30-55 GHz. One band can be used for sensing, while an additional communication channel can be established in the other band and used for sending "wake-up" signals to minimize the power consumption. Due to the ease of scalability of this resonant structure, the authors have fabricated a prototype that operates in the range of $5-8 \mathrm{GHz}$ as a proof-of-concept to demonstrate how the sensor works.

\section{RF TRANSDUCER DESIGN AND OPERATION PRINCIPLES}

The topology of the sensor structure is shown in Figs. 1 and 2. The top view of the stacked patch resonator, shown in Fig. 1 , indicates a square patch positioned right above another square patch, both are centered on top of the grounded cavity. The Si membrane is not shown in Fig. 1. The side view of the $\mathrm{RF}$ sensor is shown in Fig. 2. It is shown here that the upper patch is attached to the bottom of the Si membrane that seals over an air cavity. The cavity is surrounded by metalized walls that are grounded. The lower patch is placed on top of the first LTCC substrate using GL550 $\left(\mathrm{eps}_{\mathrm{r}}=5.6\right)$ by Kyocera Corp. The second substrate, identical to the first one, is placed underneath the ground plane to support a microstrip line $(50 \Omega)$ that feeds the lower patch through a signal via that passes through a hole with size ' $\mathrm{g}$ ' in the ground (Fig. 2). In fabrication, the metalized walls can be realized in fabrication 
by using vias [3], and the Si membrane may be supported by additional LTCC layers as a frame for the air cavity.

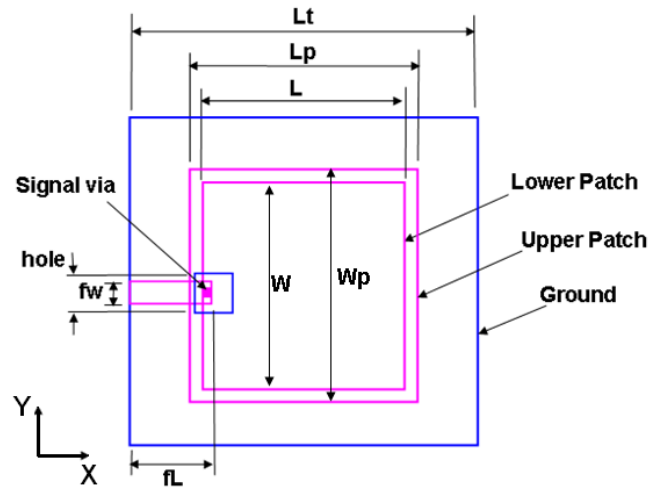

Fig. 1. Top view of the RF pressure transducer.

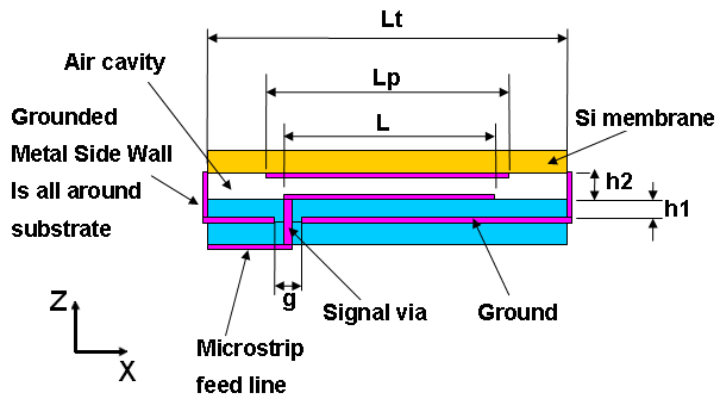

Fig. 2. Side view of the RF pressure transducer.

The major parameters for the RF transducer designed for 30-55 GHz range are follows: $\mathrm{Lt}=3340$ um (total length of the square ground), $\mathrm{Lp}=\mathrm{Wp}=1800 \mathrm{um}$ (length and width of the upper patch, respectively), $\mathrm{L}=\mathrm{W}=1670$ um (length and width of the lower patch, respectively), h1 (thickness of LTCC substrate $)=82 \mathrm{um}$, and h2 (air gap) $=40-100 \mathrm{um}$.

The dual-band performance of the stacked-patch resonator is due to the presence of the two close resonant frequencies introduced by the two patches. The upper patch's resonance is created through coupling between the upper and lower patches through the air gap h2, which can be modeled by the equivalent circuit shown in Fig. 3 [4], in which the two patches are modeled as two parallel resonant circuits with $\mathrm{R}_{1}$, $\mathrm{R}_{2}$ representing the radiation resistances for each patch, $\mathrm{L}_{1}$, $\mathrm{L}_{2}$ and $\mathrm{C}_{1}, \mathrm{C}_{2}$ representing the equivalent inductances and capacitances, respectively, for each patch, and $\mathrm{M}$ and $\mathrm{C}$ corresponding to the coupling inductance and capacitance, respectively. (Subscript 1 and 2 refer to the lower and upper patch, respectively.) The two resonant frequencies depend on $\mathrm{L}_{1} \mathrm{C}_{1}$ and $\mathrm{L}_{2} \mathrm{C}_{2}$, while the coupling effectiveness is due to the coupling capacitance $\mathrm{C}$ and mutual inductance $\mathrm{M}$ [4].

From the surface current analysis, it is found that the higher resonance is due to the lower patch, while the lower one is due to the upper patch. Therefore, as $\mathrm{h} 2$ varies, $\mathrm{C} 1$ parameter is directly affected, which consequently shifts the higher resonant frequency while the change in $\mathrm{C}$ only alters the coupling effect leaving $\mathrm{C} 2$ almost the same, thus resulting in a change in the return loss of the lower frequency but not a frequency shift. Since the upper patch is attached to the $\mathrm{Si}$ diaphragm, the deflection of the diaphragm due to pressure changes directly corresponds to changes in $\mathrm{h} 2$, which is responsible for the frequency shift. Therefore, the stackedpatch resonator functions as a transducer that transforms the difference in pressure between the air cavity and that of the surrounding environment into a shift of resonant frequency for detection.

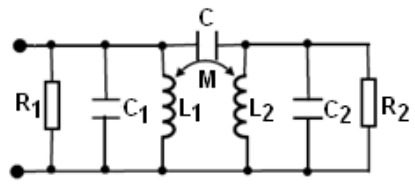

Fig. 3. Equivalent circuit of the stacked-patch transducer [4].

\section{SIMULATIONS, SCALED PROTOTYPE, AND MEASUREMENTS}

The deflection of the $\mathrm{Si}$ membrane due to differential pressure is first approximated with uniform displacements of both the upper patch and the Si layer, concurrently. Then, the approximation is improved with curved surface deflections as observed in a real physical membrane. These approximations are simulated in MicroStripes 7.1 by Flomerics Inc.

\section{A. Simulations of the RF pressure transducer at 30-55 GHz}

The return loss of the stacked-patch resonator (in Figs. 1 and 2) is shown in Fig. 4. Here, a dual-band frequency response is observed. The resonant frequency in the higher band is shifted from 47.6 to $54.6 \mathrm{GHz}$ as the distance h2 between two patches decreases from 100 to $40 \mathrm{um}$. The lower resonant frequency remains almost constant during this range of displacement. Thus, on average the RF transducer design shows a high sensitivity of $116 \mathrm{MHz} / \mathrm{um}$ which is directly detectable in a remote sensing / radar system. It is important to note that although the higher band resonant frequency shifts as h2 changes, the lower band resonant frequency (at $32 \mathrm{GHz}$ ) is unaffected by the change. Therefore, a reliable wireless communications link can be established at the lower band.

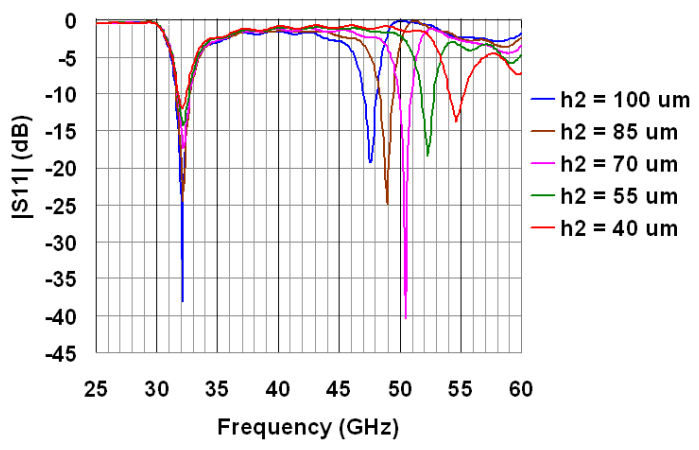

Fig. 4. Return loss of LTCC based RF pressure transducer with a) h2 = 100 um (blue line), b) h2 = 85 um (brown line), c) h2 = 70 um (pink line), d) h2 = 55 um (green line), and e) h2 = 40 um (red line). 
B. Design of the 5-8 GHz band prototype and improved approximation of the Si membrane defection

Without loss of generality and as a simple proof-of-concept, the stacked-patch resonator was scaled to operate between 5-8 $\mathrm{GHz}$ and was simplified by replacing the microstrip feedline with a probe feed; hence, the bottom substrate layer below the ground (Fig. 2) is removed. The new scaled design is shown in Fig. 5 with $\mathrm{Lt}=\mathrm{Wt}=32 \mathrm{~mm}$ (total size of the ground), $\mathrm{Lp}=$ $\mathrm{Wp}=19 \mathrm{~mm}$ (size of the upper patch), and $\mathrm{L}=\mathrm{W}=17 \mathrm{~mm}$ (size of lower patch). The feed point is placed at the center along the radiating edge of the lower patch. The lower patch LTCC substrate and upper patch superstrate (top $\mathrm{Si}$ membrane), shown in Fig. 2, are replaced by layers of $\mathrm{RT} /$ duroid 5880 substrate with $\mathrm{eps}_{\mathrm{r}}=2.2$. The lower patch substrate thickness is $1575 \mathrm{um}$, and the superstrate thickness (colored yellow in Fig. 5 with the upper patch placed underneath) is $787 \mathrm{um}$.

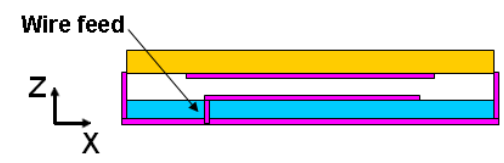

Fig. 5. Top view of the $6 \mathrm{GHz}$ design of RF pressure transducer with coaxial feeding.

The improved approximation of the membrane deflection is modeled by a surface using spherical curvature of radius $\mathrm{R}$ as indicated in Fig. 6 . The deflection is denoted by $\Delta \mathrm{h}$, and $\mathrm{Ld}$ is the diagonal length of the square grounded cavity. Angle $\theta$ is chosen such that the curved surface does not exceed the tensile stress tolerance specified in the RT/duroid 5880 material data sheet. Parameters R and $\theta$ are varied to obtain the desired $\Delta \mathrm{h}$. A 3D illustration of both the uniform displacement surface and the curved surface approximations of membrane deflection are shown in Fig. 7. Plots of the simulated return loss of the two approximation models are shown in Figs. 9 and 10 , respectivelv.

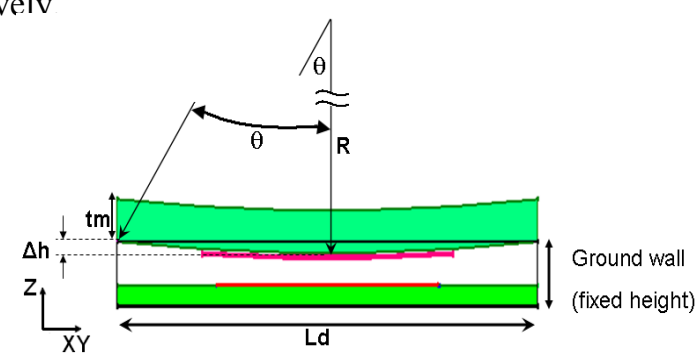

Fig. 6. Spherical curvature modeling for the improved approximation of membrane deflection.

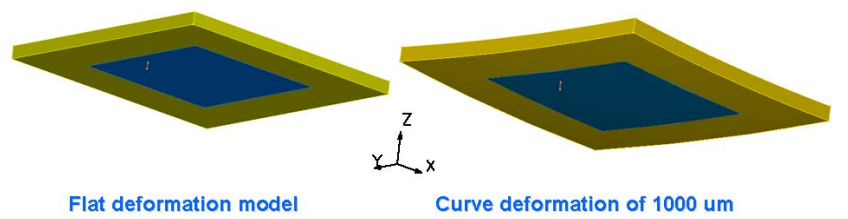

Fig. 7. 3D illustration of the two approximations to model the deflection of the membrane in the RF pressure transducer.
The plots in Figs. 8 and 9 indicate that the uniform displacement approximation of the diaphragm is sufficient in modeling the working of the stacked-patch RF pressure transducer. It should be noted that the plot in Fig. 9 shows simulations of 200 um deflection interval, while that in Fig. 8 shows a deflection interval of $250 \mathrm{um}$ in h2. Therefore, without compromising the quality of the RF pressure transducer, this prototype was fabricated in a way that the thickness of the superstrate (supporting the upper patch) is mechanically adjusted to represent the uniform displacement of the membrane in the transducer.

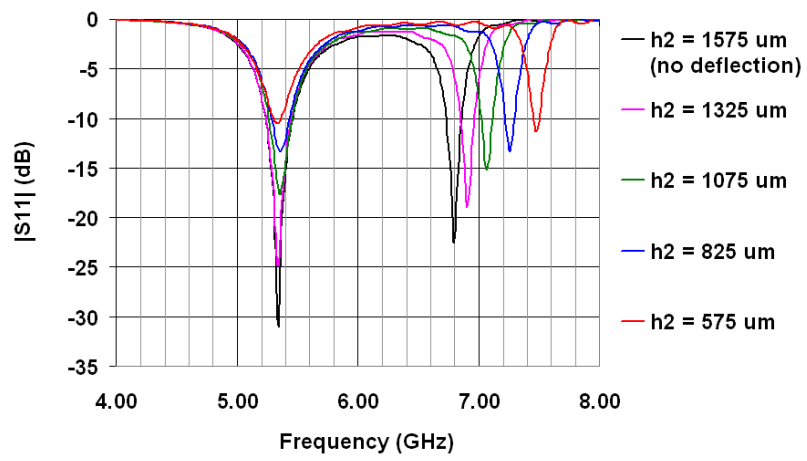

Fig. 8. Simulated return loss of $6 \mathrm{GHz}$ scaled RF transducer design of uniform displacement for membrane deflection with a) h2 $=1575$ um (black line), b) h2 $=1325$ um (pink line), c) h2 = 1075 um (green line), d) h2 $=825$ um (blue line), and e) $\mathrm{h} 2=575 \mathrm{um}$ (red line).

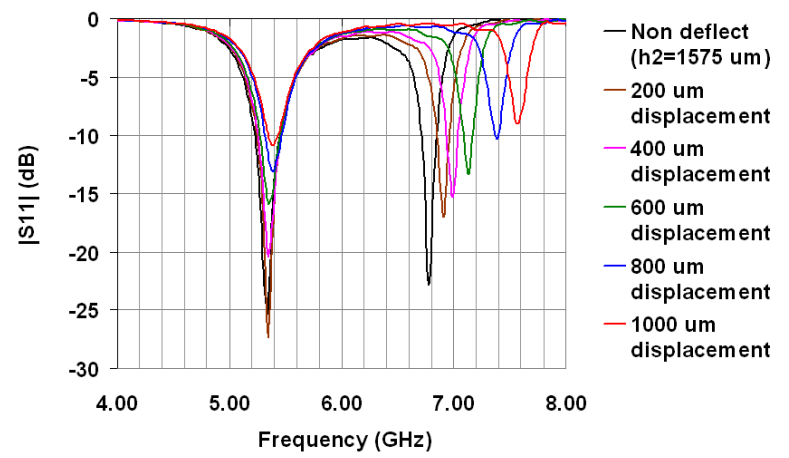

Fig. 9. Simulated return loss of $6 \mathrm{GHz}$ scaled RF transducer design of curvature improved approximation for membrane deflection with a) $\Delta \mathrm{h}=0$ (black line), b) $\Delta \mathrm{h}=200 \mathrm{um}$ (brown line), c) $\Delta \mathrm{h}=400 \mathrm{um}$ (pink line), d) $\Delta \mathrm{h}=600 \mathrm{um}$ (green line), e) $\Delta \mathrm{h}=800 \mathrm{um}$ (blue line), and f) $\Delta \mathrm{h}=1000 \mathrm{um}$ (red line).

\section{Fabrication of the 5-8 GHz band prototype RF transducer and measurement results}

The prototype was fabricated using Quick Circuit Systems of T-Tech Inc. The fabricated structure is illustrated in Fig. 10 indicating how the layers are positioned in the prototype. The resonator prototype is excited with a coaxial probe feed, and the buffer layers between the upper and the lower patches use 
FR4 in order to mechanically form an air cavity between the upper and lower patches. In the prototype, since the probe feed introduces additional inductance added to L1 (section II), dimension $\mathrm{L}$ is shortened by $2 \mathrm{~mm}$ with respect to $\mathrm{W}$ (Fig. 1) in order to match to the coaxial line $(50 \Omega)$.

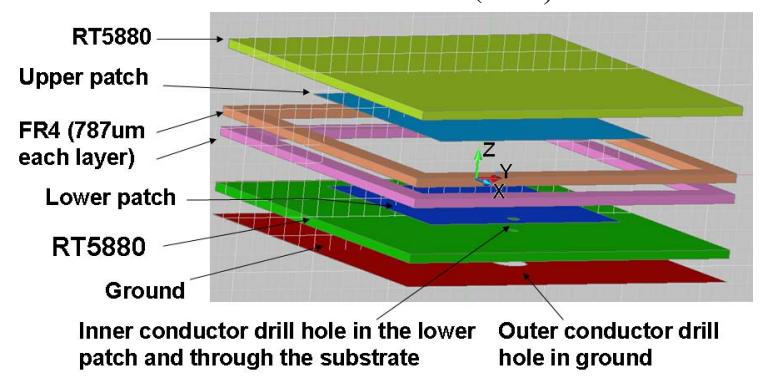

Fig. 10. The exploded view of the fabricated prototype of the $6 \mathrm{GHz} \mathrm{RF}$ transducer scaled design.

Separated fabricated layers as well as the assembled prototype are shown in Fig. 11. The layers are held together using plastic fasteners that allow the removal of the FR4 frames. The height of the air cavity is determined by the number of FR4 layers stacked between the upper and lower patches. The prototype is assembled with two different air gap thicknesses (h2) of $787 \mathrm{um}$ and $1575 \mathrm{um}$ corresponding to 1 and 2 layers of FR4. The grounded metalized walls surrounding the air cavity are constructed using copper tape at the outer edges of the prototype covered to the top of the FR4 layers only (Fig. 11). The measurement results of the return loss of these two thicknesses are plotted in Fig. 12.

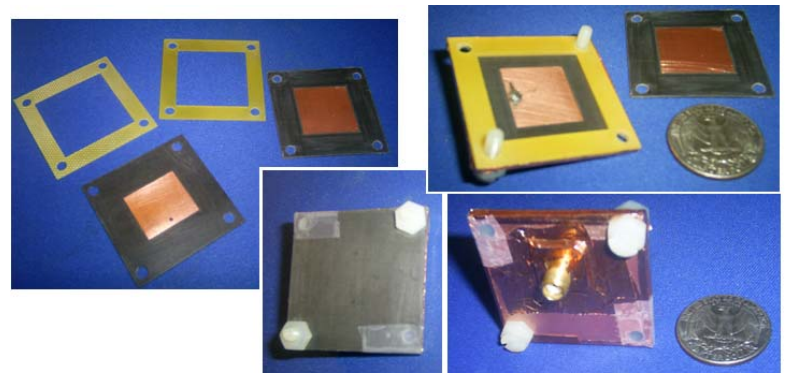

Fig. 11. Separate fabricated layers and assembled prototype.

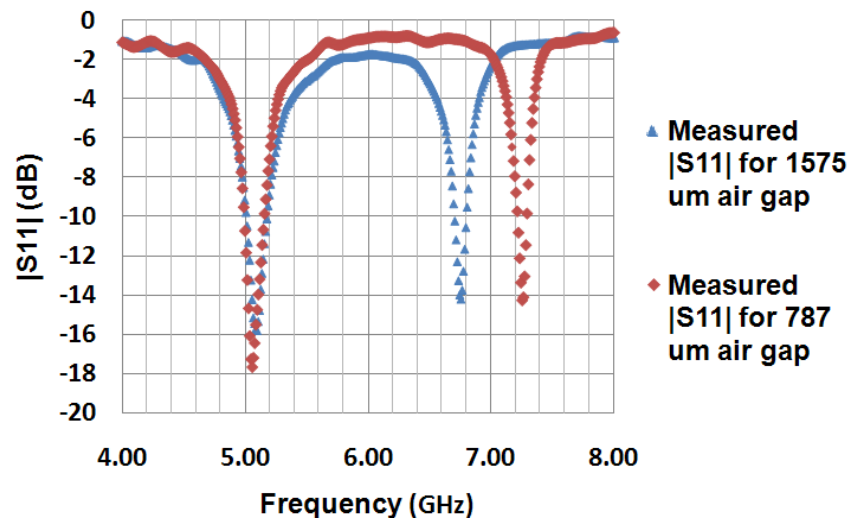

Fig. 12. Measurement results of return loss of the $6 \mathrm{GHz}$ prototype of the scaled RF pressure transducer design.
The results shown in Fig. 12 are in excellent agreement with the simulated results shown in Figs. 8 and 9. A shift of approx. $250 \mathrm{MHz}$ (due to the height difference of $787 \mathrm{um}$ ) is recorded in the measurements. Thus, as proof-of-concept, the prototype has successfully demonstrated the ideas and operability of the RF pressure transducer proposed in this paper.

\section{CONCLUSION}

A new highly sensitive radio frequency pressure transducer has been designed to operate in the millimeter wave frequency range that can be seamlessly integrated with other RF circuits in the LTCC multilayer packaging technology. Furthermore, the RF transducer presented here, operating in two frequency bands between $30-55 \mathrm{GHz}$, serves as both a wireless communications link and a remote sensing differential pressure indicator. This device can simultaneously simplify the design process, reduce the device's size, and reduce power consumption of the sensor at device level, providing a sensitivity of $116 \mathrm{MHz} / \mathrm{um}$ with respect to the diaphragm deflection due to pressure change. The operational principles of the transducer design have been successfully demonstrated through a proof-of-concept prototype operating between 5-8 $\mathrm{GHz}$ that shows a frequency shift of $250 \mathrm{MHz} / 787 \mathrm{um}$. In future work, the millimeter wave prototype based on LTCC using $\mathrm{Si}$ membrane will be fabricated that responses to physical pressure change. The design can also be modified to the use multilayer organics, such as LCP that allow realization of wireless implantable sensors for biomedical applications.

\section{ACKNOWLEDGEMENT}

The authors are grateful to T-Tech Inc., whose assistance were essential to the realization of the prototype presented here, and Drs. Jim Kajiya and Turner Whitted for their helpful insights that led to this research. In addition, the authors would also like to acknowledge the support of Georgia Electronic Design Center on this work.

\section{REFERENCES}

[1] P. Kolinko, and L. E. Larson, "Passive RF Receiver Design for Wireless Sensor Networks," IEEE/MTT-S International Microwave Symposium, pp.567-570, June 2007.

[2] M. M. Jatlaoui, and P. Pons, H. Aubert, "Pressure Micro-sensor based on Radio-Frequency Transducer," IEEE/MTT-S International Microwave Symposium, pp.1203-1206, June 2008.

[3] B.Pan, Y.Li, M.M.Tentzeris and J.Papapolymerou, "A High-Q Millimeter-Wave Air-Lifted Cavity Resonator on Lossy Substrates," IEEE Microwave and Wireless Components Letters, Vol.17, No.8, pp.571-573, August 2007.

[4] R.L. Li, G. R. Dejean, K. Lim, M. M. Tentzeris, and J. Laskar, "Design of Compact Stacked-Patch Antennas in LTCC Multilayer Packaging Modules for Wireless Applications,' IEEE Trans. Advanced Packaging, Vol.14, No.7, pp.361-363, July 2004. 